\title{
A produção científica brasileira sobre o Projeto Mais Médicos para o Brasil (PMMB)
}

\author{
The Brazilian scientific production on More Doctors Project for Brazil \\ (PMMB)
}

Quelen Tanize Alves da Silva', Luiz Carlos de Oliveira Cecílio'

DOI: $10.1590 / 0103-1104201912121$

RESUMO Esse estudo configura-se como uma pesquisa bibliográfica do Programa Mais Médicos, especificamente, sobre a produção científica referente ao Projeto Mais Médicos para o Brasil, o eixo de provimento emergencial de profissionais. Para tanto, foi realizada busca na Biblioteca Virtual em Saúde, nos bancos de dados da Medline, Lilacs e SciELO, no Banco de Teses e Dissertações da Capes e na Plataforma de Conhecimento do Programa Mais Médicos, organizada pela Rede de Atenção Primária em Saúde da Associação Brasileira de Saúde Coletica (Abrasco). O descritor usado foi 'Programa Mais Médicos em Saúde’. Os dados demonstram um esforço em análises do Programa a partir dos espaços locais onde se desenvolvia a política, o que permite a compreensão da implementação do Programa, nas diferentes regiões do País. No entanto, verificou-se pouca produção de conhecimentos e pesquisas advindas dos serviços, sendo realizadas, em grande parte, pelas Instituições de Ensino Superior. Nesse estudo, verificou-se o interesse de diferentes áreas de conhecimento, o que permite a construção de caminhos para uma análise crítica da política em curso, rompendo com a ideia que apenas um campo específico tem capacidade de construir conhecimento sobre política.

PALAVRAS-CHAVE Política de saúde. Planos e programas de saúde. Emprego. Revisão.

1 Universidade Federal de São Paulo (Unifesp) - São Paulo (SP), Brasil. qdasilva@yahoo.com.br
ABSTRACT This study is a bibliographical research of the Mais Médicos Program, specifically, on the scientific production referring to the Project More Doctors for Brazil, the axis of emergency provision of professionals. To that end, a search was made for the Virtual Health Library, the Medline, Lilacs and SciELO databases, the Capes Thesis and Dissertations Bank and the Doctors' Knowledge Platform, organized by the Primary Health Care Network of the Brazilian Association of Public Health (Abrasco). The descriptor used was 'Program More Doctors in Health'. The data demonstrate an effort in analyzes of the Program from the local spaces where the policy was developed, which allows the understanding of the implementation of the Program in the different regions of the country. However, there was little production of knowledge and research resulting from the services, and was largely carried out by Higher Education Institutions. In this study, the interest of different areas of knowledge was verified, which allows the construction of ways for a critical analysis of the current policy, breaking with the idea that only a specific field has the capacity to build knowledge about politics.

KEYWORDS Health policy. Health programs and plans. Employment. Review. 


\section{Introdução}

O Programa Mais Médicos (PMM) foi lançado como resposta a demandas sociais em um momento de crise, as quais culminaram em manifestações de rua em junho e julho de 2013: as chamadas 'Jornadas de Junho'. Dentre as pautas desse movimento social, destacava-se a má qualidade dos serviços públicos. Nesse sentido, mais uma vez, observa-se que, apesar das evidências de pesquisas, as pressões políticas de vários grupos de lobby e da mídia induzem os tomadores de decisão a apresentar respostas rápidas em um curto período na formulação de uma política ${ }^{1}$.

Ainda em 2013, novos prefeitos e vereadores iniciavam seus mandatos, protestando contra a falta de médicos nos municípios localizados em regiões de baixa atratividade para esses profissionais. Esse movimento constituiu a Marcha dos Prefeitos, que tinha como tema 'Cadê o Médico?'. Dessa forma, o PMM foi constituído a partir da articulação de ações em fase de planejamento ou de execução que, no conjunto, procuravam dar respostas de curto, médio e longo prazo aos problemas enfrentados pela área da saúde. Embora, no seu conjunto, seja um programa novo, as soluções que o compõem já estavam formuladas separadamente, esperando apenas o momento ideal para serem implementadas (ou seja, a abertura de uma janela de oportunidade) a partir de um novo arranjo ${ }^{2}$.

Estudos apresentam relatos de sujeitos, informantes-chave, que comentam sobre uma reunião nacional de secretários municipais de saúde, ocorrida em 2012, na região Nordeste. $\mathrm{O}$ intuito era debater com a Presidente Dilma Rousseff sobre a dificuldade de montar a rede de atenção básica na região devido à dificuldade de preenchimento das vagas de médicos em concursos públicos ${ }^{3}$.

A ampliação do acesso e a melhoria nas áreas de saúde já vinham sendo debatidas e programadas no interior do Governo Dilma Rousseff. Verificou-se que o Governo vinha planejando, desde janeiro de 2012, ações na saúde pública que buscassem responder à demanda por profissionais médicos. Em visita oficial a Cuba, a presidente Dilma iniciou negociações para o envio dos médicos cubanos para o Brasil ${ }^{4}$. Esse cenário, potencializado pelas manifestações de junho de 2013, teria possibilitado a construção e o lançamento do PMM, um programa de provimento médico que, segundo o Ministério da Saúde, permitiria efetivas mudanças para o fortalecimento da Atenção Básica em Saúde (ABS) no País, garantindo maior acesso da população aos serviços de saúde 5 .

O PMM foi lançado em julho de 2013 com a Medida Provisória ${ }^{\circ}$ 621, posteriormente convertida na Lei $\mathrm{n}^{\circ}$ 12.871, em outubro de 2013. Foi baseado em evidências que apontavam para um cenário de escassez de médicos no País, sobretudo na Atenção Primária à Saúde (APS).

O PMM foi estruturado em três eixos: a) Infraestrutura da Atenção Básica em Saúde; b) Organizacional ou Educacional; e c) Provimento Emergencial, denominado Projeto Mais Médicos para o Brasil (PMMB), este último com os seguintes objetivos: diminuir a carência de médicos nas regiões prioritárias; reduzir as desigualdades no acesso à saúde; fortalecer a ABS; aprimorar a formação médica; ampliar a inserção do médico em formação na ABS; fortalecer a política de educação permanente com a integração ensino e serviço; promover troca de conhecimentos e experiências entre profissionais de saúde brasileiros e médicos formados no exterior; aperfeiçoar a formação de médicos para atuar nas políticas públicas do Sistema Único de Saúde (SUS) e estimular pesquisas relacionadas com o SUS. A intencionalidade dessa ação era atender à população de forma imediata, até que as ações com foco na ampliação da formação do médico expressassem resultados ${ }^{6}$.

A grande inovação do PMM se refere à estratégia de chamadas internacionais: além dos médicos brasileiros, o programa passou a contar com médicos estrangeiros de 49 países. Dos médicos estrangeiros que atuaram no PMM, a maioria era de cubanos, 
recrutados por meio de uma parceria entre o Ministério da Saúde do Brasil e a Organização Pan-Americana da Saúde e, desta última, com o Ministerio de Salud Pública de Cuba ${ }^{7}$.

A partir de recomendações da Organização Mundial da Saúde (OMS), o recrutamento de médicos estrangeiros se restringiu àqueles que atuavam em países com proporção de profissionais maior que a do Brasil. Foram selecionados os médicos graduados em instituições reconhecidas por seus países e cuja formação curricular fosse adotada no Brasil e mundialmente reconhecida ${ }^{2}$.

O Governo Federal lançou um edital público para que médicos se alistassem para atuar nas Unidades Básicas de Saúde (UBS) nas regiões prioritárias do SUS, e para adesão de municípios elegíveis ao Programa. Foram aceitas a participação de médicos formados no Brasil, bem como a de graduados em outros países. Esses profissionais tinham a supervisão de uma universidade e, durante o período de participação no programa, realizavam um curso de especialização em atenção básica. Os médicos estrangeiros passavam por quatro semanas de treinamento intensivo oferecido pelas universidades brasileiras, que aborda linguagem clínica, os protocolos assistenciais nacionais e o SUS. Os aprovados recebiam/recebem um registro provisório para exercício da medicina, chamado Registro Único do Ministério da Saúde (RMS). Foi criado um visto especial para médicos participantes do PMM, emitido pelo Ministério das Relações Exteriores ${ }^{7}$.

Todos os municípios brasileiros poderiam/ podem participar do PMM; no entanto, não é permitido substituir os médicos já atuantes na Rede. Os critérios de prioridade para ocupação das vagas, de acordo com os documentos do programa, são: municípios com percentual de população em extrema pobreza igual ou superior a 20\%, com Índice de Desenvolvimento Humano (IDH) baixo/muito baixo, e integrante de regiões prioritárias; regiões de alta vulnerabilidade de capitais, regiões metropolitanas e grupo de municípios populosos, mas com baixa arrecadação; e Distritos Sanitários Especiais Indígenas².
Ficam sumarizadas, assim, algumas dimensões em jogo nessa política pública, cujo escopo caminha na construção de um sistema nacional de saúde plasmado pelo princípio da universalização da assistência à saúde como direito de cidadania e dever do Estado. Desse sistema, derivam estratégias como a construção de uma atenção básica coordenadora dos esforços de integração do cuidado em todos os níveis, adequada às necessidades de saúde da população assistida, tanto do ponto de vista tecnológico como do ponto de vista da humanização de suas práticas.

Nessa perspectiva, o PMMB (eixo provimento emergencial) constitui-se como uma de suas ações mais mobilizadoras do Programa, tanto daqueles que o apoiaram quanto dos que o hostilizaram. Sob essa perspectiva, abre-se a necessidade de estudos que possam subsidiar e produzir evidências explicativas e compreensivas dos impactos dessa política governamental. Dessa forma, a presente revisão foi focada no eixo provimento emergencial, denominado PMMB.

\section{Consolidar o SUS: a dimensão técnica do programa}

O Brasil, no ano de 2013, possuía 359.691 médicos ativos, e apresentava uma proporção de 1,8 médico para cada mil habitantes, conforme dados primários obtidos no Conselho Federal de Medicina (CFM) e na estimativa populacional do Instituto Brasileiro de Geografia e Estatística (IBGE). A proporção de médico/ mil habitantes constatada no Brasil era menor do que em outros países latino-americanos com perfil socioeconômico semelhante ou em países que têm sistemas universais de saúde ${ }^{6,8}$. Essas evidências indicavam um número insuficiente de médicos por habitante em comparação a outros países, má distribuição no território brasileiro, dificuldade de atração e fixação de profissionais em regiões de necessidade e uma percepção social de que essa carência era o principal problema do SUS. 
Além disso, na última década, os salários dessa categoria mostraram-se crescentes, em um cenário caracterizado pela maior formalização dos postos de trabalhos?.

Soma-se aos estudos supracitados uma pesquisa de opinião nacional, que, em 2011, revelou que $58 \%$ dos respondentes apontaram a 'falta de médicos' como o principal problema do SUS10. Existe, portanto, uma lacuna entre o SUS constitucional e o SUS real. A intencionalidade de saúde como um direito de todas e todos e da universalidade do acesso aos serviços de saúde, ainda hoje, são desafios a serem alcançados.

A força de trabalho é fundamental para o fortalecimento dos sistemas de saúde. Vários países vêm se esforçando para formar profissionais em quantidade e com as qualidades necessárias para suprir as necessidades de saúde de suas populações. Esses esforços nem sempre atingem resultados esperados, devido à migração de trabalhadores para outros países, seja pela remuneração, seja por melhores condições de vida ou pela estabilidade política, entre outras possíveis causas ${ }^{\mathbf{1 1} 12}$. O prejuízo causado pela perda de trabalhadores qualificados é evidente quando se verifica que os sistemas educacionais de muitos desses países não são capazes de manter um quadro profissional estável para a formação futura de outros profissionais da área ${ }^{13}$.

O êxodo de profissionais de regiões menos desenvolvidas e mais pobres para outras com melhores condições de vida constitui-se em um problema para muitos países, provocando concentrações em algumas áreas e déficit em outras. A crise de profissionais da saúde é cada vez mais importante nas agendas dos países desenvolvidos e em desenvolvimento, caracterizando obstáculo para o fortalecimento dos sistemas nacionais de saúde 14-16.

Em todos os países, ações relativas ao provimento e à fixação de profissionais de saúde são de responsabilidade do Estado, principalmente naqueles com serviços de saúde universais e gratuitos, uma vez que as áreas de vulnerabilidade de saúde também são as áreas de vulnerabilidade social, econômica, desintegradas do restante do País ${ }^{13,19}$.

É importante referir que esses elementos têm sido objeto de estudos e pesquisas, transformando a temática de 'recursos humanos em saúde' em área específica de investigação acadêmica e científica. Todavia, as tentativas de reordenamento dessa área, seja no âmbito da formação, seja nas questões relativas ao trabalho, ainda não foram capazes de responder efetivamente aos desafios colocados. Se, por um lado, avançam as análises, propostas e estudos sobre o tema, por outro, permanece o hiato em incorporá-los à ação política efetiva ${ }^{20,21}$.

Naúltima década, diferentes países têm focado seus esforços no fortalecimento dos sistemas de proteção social e na cobertura universal dos serviços de saúde ${ }^{17,18}$. Nesse contexto, de maneira estratégica, tem sido priorizado o desenvolvimento e fortalecimento da atenção primária. Organizar a melhor disponibilidade e a distribuição de trabalhadores constitui aspectos relevantes para atingir os objetivos pretendidos de resolutividade da assistência em saúde ${ }^{19}$. No entanto, para organização de um sistema de saúde em redes integradas e baseado em princípios de APS, apesar dos avanços significativos observados, é necessário enfrentar diferentes e diversos desafios, especificamente no âmbito de gestão de trabalho da saúde 22 .

Há escassez de profissionais, principalmente médicos, em várias regiões do País. A Estratégia Saúde da Família (ESF) e os serviços de urgência (portas de entrada do Sistema) são grandes desafios a serem enfrentados no provimento e fixação de profissionais de saúde em todas as regiões do País ${ }^{19}$.

No Brasil, nos últimos anos, tomaram força as discussões sobre a força de trabalho em saúde a partir do advento da ESF, que trouxe consigo inúmeros desafios a esse campo, como a carência de profissionais de saúde em diversas regiões e o descompasso entre o ensino e as necessidades da atenção básica.

A ESF é considerada a forma prioritária para reorganização da APS, conforme preceitos do SUS. Anteriormente denominado Programa Saúde da Família, foi criado, em 1994, 
com o objetivo de reorganizar a atenção básica tanto no que se refere às demandas populacionais quanto no que se refere às práticas de saúde ${ }^{23}$. A ESF é tida pelos gestores dos diferentes âmbitos governamentais como uma estratégia de expansão, qualificação e consolidação da ABS para favorecer uma reorganização de processos de trabalho, ampliando a resolutividade e o impacto na situação de saúde das pessoas e coletividades ${ }^{19}$.

Entre as questões da força de trabalho em saúde, a necessidade de adequar o provimento de serviços de saúde nas regiões remotas, periféricas e de maior vulnerabilidade tem sido identificada como um dos nós críticos para o sucesso da ESF. O Brasil avançou bastante nas últimas décadas nessa área; porém, ainda existem muitos vazios assistenciais que correspondem a localidades que não conseguem prover e fixar profissionais de saúde na atenção básica, em especial, os médicos, não garantindo acesso aos serviços básicos de saúde por parte da população brasileira ${ }^{19}$.

As vagas desocupadas nos serviços de saúde evidenciam a falta de profissionais, fator que existe tanto nos países ricos como nos pobres. A escassez de profissionais da saúde, especificamente, os médicos, é mais acentuada em áreas remotas e socioeconomicamente vulneráveis ${ }^{24}$. Assim, torna-se necessário interferir nos condicionantes que permitam a estruturação e a organização desse nível de atenção em saúde. A alta rotatividade dos médicos nas equipes de saúde da família continua sendo um problema nos grandes centros urbanos ${ }^{25}$, ainda que estudos recentes indiquem um índice de permanência maior em relação ao início da estratégia; a rotatividade de profissionais, principalmente os médicos, colocam-se como entraves ao desenvolvimento do trabalho ${ }^{26}$.

\section{Métodos}

Este estudo se configura como uma pesquisa bibliográfica da produção teórica brasileira referente ao PMM, especificamente aquela que se ocupa do PMMB, ou seja, o provimento emergencial de médicos naquelas localidades mais desassistidas. A revisão aqui proposta é parte de uma investigação mais ampla, a qual não teve financiamento público ou privado para o seu desenvolvimento e que tem como objetivo principal caracterizar os sentidos dados ao PMM pelos médicos que dele participam. Partiu-se do pressuposto de que haveria uma insuficiência na produção teórica brasileira de trabalhos que adotem tal perspectiva, qual seja: que sentidos os médicos contratados pelo programa dão a uma política como o Mais Médicos tendo em vista suas vivências locais com o programa?

A justificativa para tal interesse situa-se na perspectiva de identificar quais estudos têm abordado a dimensão mais micropolítica do PMM, contribuindo para o seu aperfeiçoamento ao incorporar a perspectiva de seus protagonistas centrais.

A revisão foi realizada com a intencionalidade de, em primeiro lugar, reconhecer estudos que se ocuparam dos elementos políticos, sociais e administrativos envolvidos nos contextos das práticas dos médicos que atuam/atuaram no PMM. Em segundo lugar, busca-se caracterizar quais estudos se ocuparam de 'dar voz' aos médicos, tentando compreender os sentidos dados por esses profissionais à sua participação no Mais Médicos, permitindo dar visibilidade às suas histórias e implicações nos contextos reais de realização da política, em uma perspectiva micro-histórica (como em Carlo Ginsburg, com sua micro-história em 'O queijo e os vermes', em 1939), ou mesmo adotado de modo brilhante por Svetlana Alexviechi, na literatura contemporânea, em sua trilogia de livros 'A guerra não tem rosto de mulher' (1935), 'Vozes de Tchernóbil' (1997) e 'O fim do homem soviético' (2016). Portanto, mesmo tendo como interesse principal os sentidos dados pelos médicos à sua participação no PMM, partiu-se do pressuposto da necessidade de uma revisão bibliográfica mais ampla, que permitisse a caracterização do contexto sócio-histórico-político do País na realização do programa. Nesse sentido, essa revisão se ocupou da produção científica brasileira produzida no período de 2013 (início do Programa) a junho de 2017. 
A revisão bibliográfica consiste em uma forma de mapear um campo do conhecimento com a finalidade de compreensão dos movimentos da área, de suas configurações, propensões teóricas metodológicas e análises críticas, indicando tendências, recorrências e lacunas ${ }^{27}$. Nessa perspectiva, a pesquisa bibliográfica é feita a partir do levantamento da produção teórica sobre determinado tema e publicada por meios escritos e eletrônicos, como livros, artigos científicos e páginas de $w e b$ sites ${ }^{\mathbf{2 8}}$.

Inicialmente, foi feita a busca das produções realizadas no Banco de Teses e Dissertações da Coordenação de Aperfeiçoamento de Pessoal de Nível Superior (Capes). Em seguida, buscou-se por pesquisas cadastradas na Plataforma de Conhecimento do PMM organizada pela Rede de Pesquisa Atenção Primária à Saúde da Associação Brasileira de Saúde Coletiva (Abrasco). Por último, houve a busca de artigos na Biblioteca Virtual em Saúde, nos bancos de dados da Medline, Literatura LatinoAmericana e do Caribe em Ciências da Saúde (Lilacs) e Scientific Electronic Library Online (SciELO). Para a seleção das publicações, foram considerados critérios de inclusão os estudos, artigos e demais publicações que citam o termo 'Programa Mais Médicos' no título; que tiveram como foco o eixo de provimento do PMM; e que foram publicados no período de 2013 a junho de 2017; que tivessem resumo e texto completo disponível em português e, especificamente, que as divulgações fossem artigos publicados em revistas.

\section{Resultados e discussões}

A busca realizada gerou 289 publicações, sendo 44 em formato acadêmico (tese ou dissertações), 40 pesquisas cadastradas e 195 artigos. Dessas produções, foram analisadas 89 publicações: 19 em formato acadêmico (tese ou dissertação), 27 pesquisas cadastradas e 43 artigos.

O gráfico 1, a seguir, apresenta a distribuição de produção científica referente ao PMMB - eixo provimento emergencial por ano nas três modalidades de produção teórica supracitadas.

Gráfico 1 - Distribuição de produção científica referente ao PMM - eixo provimento emergencial por ano

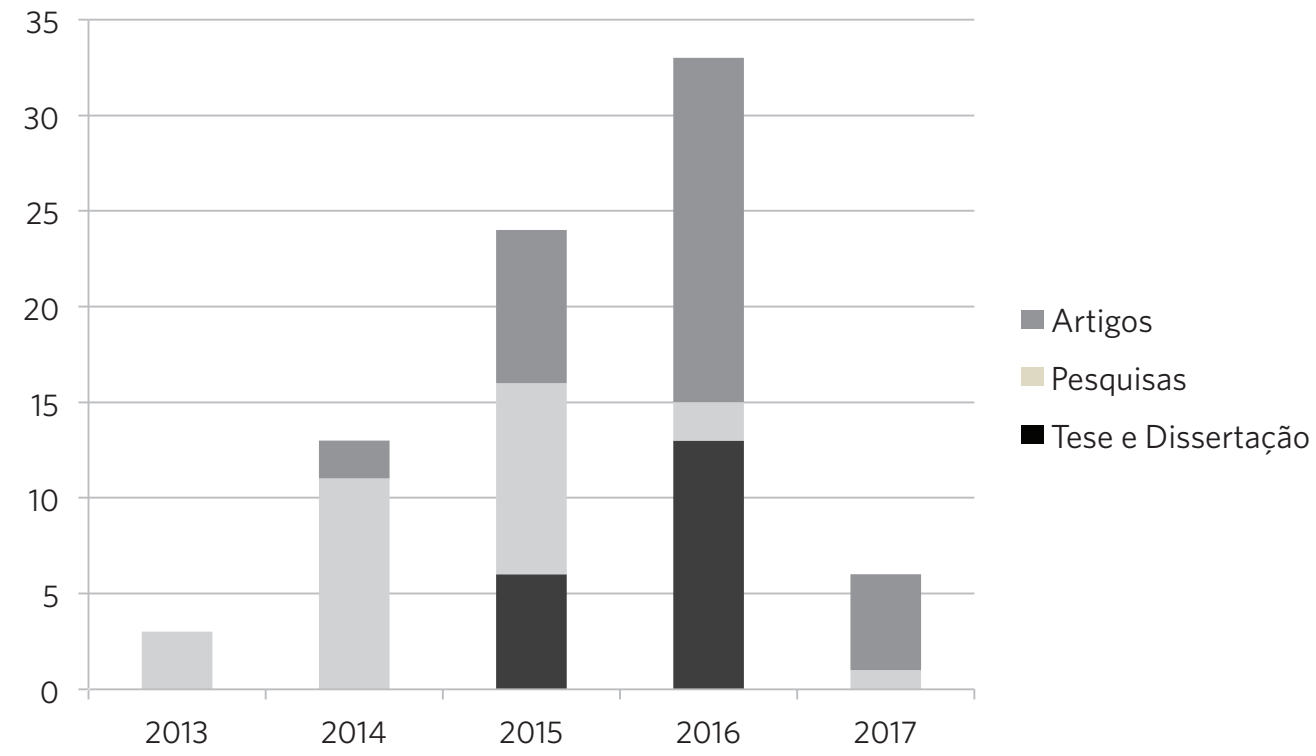


Verifica-se que, no ano de 2013, havia apenas algumas pesquisas cadastradas, quando se investiga o início de estudos cadastrados com interesse sobre o Programa. Os artigos que foram analisados no ano de 2013 foram excluídos por estarem fora do escopo da pesquisa. Muitos não possuíam resumo e quase sempre eram artigos de opinião ou debate sobre o início do Programa, caracterizando uma intensa disputa de discurso sobre a política. Em contrapartida, o ano de 2016 corresponde ao período de maior produção científica sobre o Programa.
Esse fato deve-se à chamada realizada pela revista 'Ciência \& Saúde Coletiva' para uma edição temática, alusiva aos três anos do PMM, organizada pelo Comitê Coordenador da Rede de Pesquisa em APS da Abrasco em parceria com a Organização Pan-Americana da Saúde. Constata-se, dessa forma, uma intensa produção teórica.

Em se tratando da análise da distribuição dessa produção científica em formato de artigo, apresenta-se a distribuição nas regiões do País, conforme gráfico 2.

Grafico 2. Distribuição da produção científica do PMMB - eixo emergencial por região

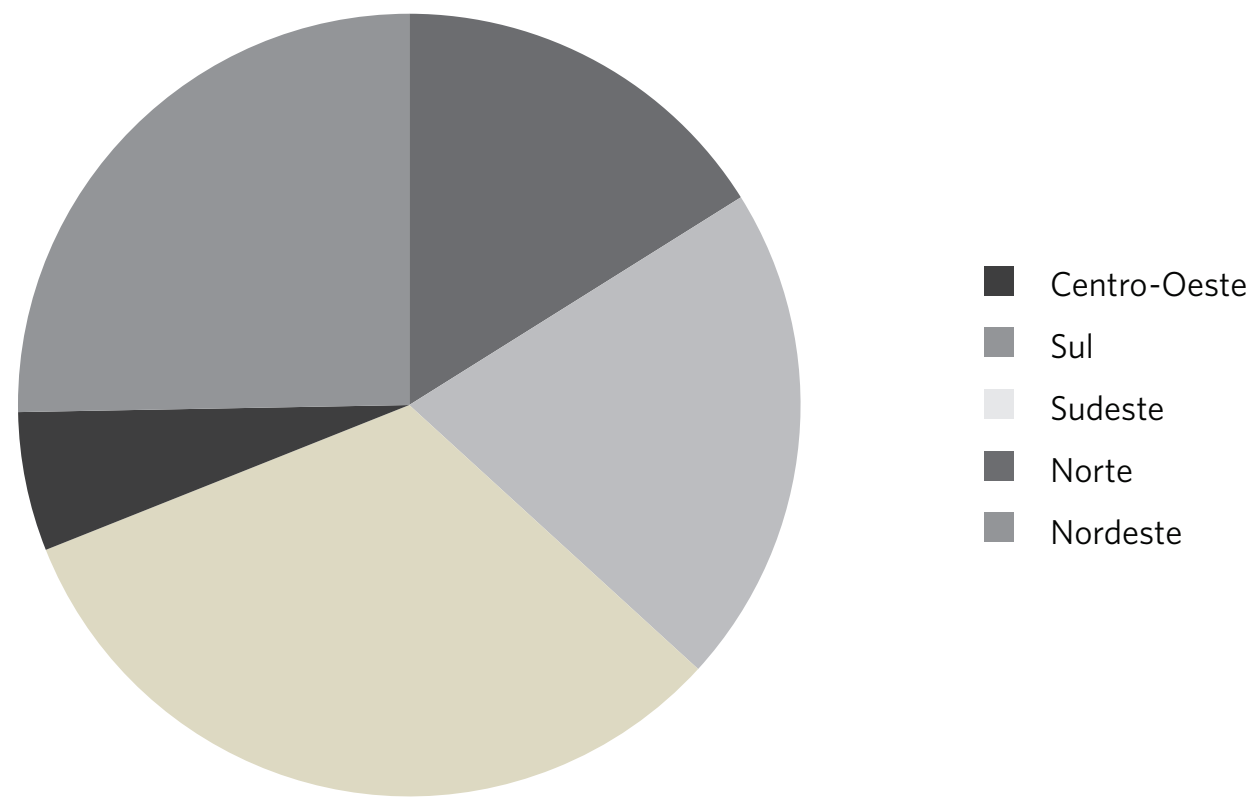

Verifica-se que a maior produção científica está localizada na região Sudeste, com 28 estudos realizados (32\%). Na sequência estão as regiões Nordeste, com 22 (25\%); Sul, com 18 (21\%); Centro-Oeste, com 14 (16\%) e Norte, com $5(6 \%)$.

Das produções científicas em formatos acadêmicos cadastradas no Banco de Tese e Dissertação da Capes e dentro do escopo dessa pesquisa, 19 (84\%) eram dissertações de mestrados. As outras 3 (16\%) eram teses de doutorado. O pouco tempo de desenvolvimento do Programa pode explicar o baixo número de teses cadastradas no Banco de Teses e Dissertações da Capes, uma vez que os programas de doutorados são desenvolvidos em até 5 anos.

Nesses estudos, predominam os realizados a partir de uma abordagem qualitativa de pesquisa. São 10 estudos nesse formato (52,63\%), enquanto 4 estudos (21\%) adotaram a abordagem quantitativa; e 5 estudos $(26,31 \%)$ 
optaram pela abordagem qualiquantitativa. A distribuição dos estudos, conforme área de conhecimento, está apresentada na tabela 1 , que segue abaixo:

Tabela 1. Distribuição de pesquisas do Banco de Tese e Dissertações da Capes sobre o PMMB - eixo provimento emergencial por área de conhecimento

\begin{tabular}{lrr}
\hline Área de Conhecimento & $\mathbf{N}$ & \% \\
\hline Saúde Coletiva & 08 & $42,1 \%$ \\
Saúde da Família & 03 & $15,8 \%$ \\
Políticas Públicas & 02 & $10,52 \%$ \\
Gestão Pública & 01 & $5.26 \%$ \\
Psicologia Sociedade e Cultura & 01 & $5.26 \%$ \\
Direito & 01 & $5.26 \%$ \\
Desenvolvimento, Sociedade de Cooperação Internacional & 01 & $5.26 \%$ \\
Ciências Sociais & 01 & $5.26 \%$ \\
Saúde Pública & 01 & $5.26 \%$ \\
\hline Total & $\mathbf{1 9}$ & $\mathbf{1 0 0 \%}$ \\
\hline
\end{tabular}

Fonte: Elaboração própria.

As pesquisas cadastradas na Plataforma de Conhecimento do PMM, na Rede APS da Abrasco, podem ser classificadas de acordo com os seguintes critérios: a abordagem teórica, a fonte de financiamento, a abrangência da pesquisa e a taxonomia do PMM. Das pesquisas cadastradas na Plataforma, 27 constituíram o escopo para análise deste estudo. Quanto às fontes de financiamento das pesquisas, observa-se que grande parte das pesquisas realizadas no período do estudo foram financiadas por órgãos governamentais, perfazendo 18 (66,67\%) das pesquisas analisadas.

A distribuição das pesquisas a partir de sua abrangência considera as instâncias de gestão do SUS (municipal, estadual, regional ou nacional). Os dados demonstram um grande esforço em análises do Programa a partir dos espaços locais em que se desenvolvia essa política. Neste sentido, a distribuição das pesquisas a partir de sua abrangência acontece da seguinte forma: as de abrangência municipal e estadual tiveram, cada uma, 8 pesquisas (30\%), 7 estudos (26\%) com abrangência nacional e 4 pesquisas (14\%) de abrangência regional.
Também é possível classificar essas pesquisas de acordo com sua taxonomia, ou seja, com o enfoque na abordagem do tema. Esses são distribuídos da seguinte forma: Avaliação de Programa e Serviços, 13 (48\%); Cultura, Saúde e Cooperação, 5(18\%); Atenção Primária à Saúde, 4(15\%), Implementação do Programa, 2 (7\%); Acesso Universal à Saúde, 1 (4\%); Fixação de Médicos, 1(4\%); Formação Interprofissional, 1(4\%).

Em relação à produção em formato de artigo, com publicação em revistas científicas, os dados foram organizados obedecendo aos seguintes critérios: quanto ao tipo de instituição à qual pertence $o$ autor principal do artigo, tipo de pesquisa/abordagem e, por último, área de conhecimento responsável pela publicação do artigo; esses foram classificados conforme o Programa de Pós-Graduação ou Departamento do vínculo institucional do autor principal.

Quanto ao tipo de instituição responsável pela publicação do artigo, considerou-se a instituição com a qual o autor principal do artigo tem vínculo. Dessa forma, observou-se que, de um total de 43 artigos publicados, uma expressiva maioria, 40 
publicações, tem como autores principais pesquisadores pertencentes a Instituições de Ensino Superior; 2 publicações são referentes a pesquisadores vinculados a órgãos governamentais e, em apenas uma dessas publicações, o autor principal tem vínculo com o serviço de saúde.
Em relação à abordagem de pesquisas dos artigos, observa-se que 28 (65\%) pesquisas são qualitativas, 12 (27,9\%) quantitativas; e apenas $3(7 \%)$ adotaram o que seria considerado como abordagem qualiquantitativa. Essa produção científica distribui-se por área de conhecimento conforme a tabela 2, abaixo:

Tabela 2. Distribuição de artigos científicos sobre o PMMB - eixo provimento emergencial por área de conhecimento

\begin{tabular}{llr}
\hline Área de conhecimento & $\mathbf{N}$ & $\%$ \\
\hline Saúde Coletiva & 13 & $30 \%$ \\
Políticas Públicas & 06 & $14 \%$ \\
Direito & 05 & $12 \%$ \\
Medicina e Ciências Biomédicas & 05 & $12 \%$ \\
Administração & 03 & $7 \%$ \\
Saúde Pública & 02 & $5 \%$ \\
Enfermagem & 02 & $5 \%$ \\
Gestão do Trabalho & 02 & $5 \%$ \\
Educação & 01 & $2 \%$ \\
Medicina Social & 01 & $2 \%$ \\
Higiene e Medicina Tropical & 01 & $2 \%$ \\
Ciências Biológicas e Saúde & 01 & $2 \%$ \\
Saúde da Família e Comunidade & 01 & $\mathbf{2} \%$ \\
\hline Total & 43 & $2 \%$ \\
\hline
\end{tabular}

Fonte: Elaboração própria.

Por fim, a revisão permitiu o agrupamento da produção nas seguintes temáticas ou (categorias': 1) Direito à Saúde; 2) Análise da arena política e implementação do Programa; 3) Provimento, fixação e distribuição de profissionais; 4) Análise de impactos sociais, indicadores e acesso à saúde; 5) Mudança de práticas e modelos de saúde; e 6) Perspectiva dos atores sociais sobre o programa. A produção científica sistematizada, a partir dessas categorias, distribui-se da seguinte forma na tabela 3:

Tabela 3. Distribuição de produção científica referente ao PMMB - eixo provimento emergencial por categorias analíticas

\begin{tabular}{|c|c|c|c|c|}
\hline Categorias & Tese & Pesquisa & Artigo & $\%$ \\
\hline Direito à Saúde & 02 & 0 & 05 & $8 \%$ \\
\hline Arena Política e Implementação do PMM & 01 & 03 & 03 & $8 \%$ \\
\hline Provimento, Fixação e distribuição de profissionais & 03 & 02 & 06 & $12 \%$ \\
\hline Análise de impactos sociais, indicadores e acesso à saúde & 04 & 04 & 17 & $28 \%$ \\
\hline Mudança de práticas e modelos de saúde & 06 & 05 & 07 & $20 \%$ \\
\hline Perspectiva dos atores sociais sobre o programa & 03 & 13 & 05 & $24 \%$ \\
\hline Total & 19 & 27 & 43 & $100 \%$ \\
\hline
\end{tabular}

Fonte: Elaboração própria. 
Verifica-se que há relevante produção científica referente à análise de impactos sociais, indicadores e acesso à saúde. São 25 estudos (28\%), o que expressa um esforço de avaliação do Programa e seus efeitos, mesmo observando o pouco tempo de desenvolvimento da Política.

\section{Análise de impactos sociais, indica- dores e acesso à saúde}

Entre os comentários sobre a assistência de médicos nos municípios antes do PMM, encontravam-se a falta total desses profissionais, a alta rotatividade ou a baixa quantidade de médicos ${ }^{29}$. A ampliação da cobertura da ABS permite a ampliação de escopo de práticas das equipes, como a realização de ações de educação, prevenção e intervenção precoce de doenças, especialmente sensíveis às ações da atenção primária, no sentido de promover a melhoria de condições de saúde e impactando, dessa forma, na redução efetiva do número de internações. Estudos demonstram que o PMM possibilitou uma tendência de redução de internações sensíveis à atenção básica em menores de 5 anos $^{30}$. Esses resultados merecem aprofundamento, buscando compreender em que medida a ampliação do acesso à assistência médica contribuiu para essa redução, associando os achados a outras variáveis, tais como os indicadores econômicos, demográficos, entre outros ${ }^{31}$.

Houve evidências, em pesquisa, da similaridade de orientações e presença dos atributos essenciais e derivados da APS, tanto nas equipes de ESF que possuem profissionais do PMM quanto naquelas que não possuem médicos pertencentes ao programa. Trata-se de uma verificação relevante, uma vez que desmistifica a ideação de que os médicos do PMM não teriam preparo adequado para atuar no Brasil, principalmente os estrangeiros, em função de supostos problemas de comunicação que a barreira das línguas diferentes poderia impor ${ }^{32}$.

O PMM objetiva fortalecer o atributo longitudinalidade quando propõe a fixação de profissionais em cidades com dificuldade de manter o médico. No entanto, como o programa preconiza um prazo determinado para a disponibilidade do profissional, em longo prazo, pode evidenciar-se como uma dificuldade na orientação desse atributo à $\mathrm{ABS}$, no que se refere ao estabelecimento de vínculo dos usuários com os profissionais de saúde ${ }^{32}$.

A produção médica da atenção básica, em 2014, após um ano de desenvolvimento do programa, evidencia a contribuição do PMM para o acesso aos serviços de saúde, medidas verificadas por meio da produção de consultas, produtividade semanal, encaminhamentos médicos e atendimentos educativos das equipes da ESF. Da mesma forma, verificou-se aumento do número de consultas na população adulta e idosa, assim como maior número de atendimentos de DST/Aids e diminuição do número das consultas fora da área de abrangência ${ }^{4,31,33}$. Observou-se aumento significativo do número de encaminhamentos para os ambulatórios de média complexidade, o que se deve à ampliação do acesso à ABS, fato esperado a curto prazo ${ }^{31,34}$.

Também, com o desenvolvimento do PMM, a responsividade dos serviços de saúde obteve avaliação positiva por parte dos usuários, tendo contribuído para esses resultados o atendimento com um tempo de espera, agora, considerado razoável. No entanto, a infraestrutura dos serviços ainda é insuficiente, precisando de maior investimento para melhor equipá-los, além da necessidade de oferta de serviços de saúde de outros níveis de complexidade nos municípios e nas correspondentes regiões ${ }^{35}$.

\section{Perspectiva dos atores sociais sobre o programa}

Para muitos usuários, a vinda de médicos estrangeiros para o Brasil deve-se ao fato de que os médicos brasileiros não querem atender no SUS, preferindo atuar no setor privado, principalmente em consultórios particulares. Houve, também, percepções negativas dos usuários quanto à vinda de médicos estrangeiros, demonstrando preocupação e insegurança 
quanto à formação desses profissionais, quanto à viabilidade da comunicação e à qualidade dos serviços prestados. Em relação à comunicação, provavelmente em função de diferentes estratégias empreendidas pelas equipes e médicos participantes do Programa, poucos usuários evidenciaram dificuldade na compreensão do idioma do médico. Mesmo aqueles que declararam essa dificuldade, indicaram que ela não inviabilizava a consulta ${ }^{34}$.

Existe o reconhecimento de que o Programa contemplou as áreas mais remotas e vulneráveis. Parte da população acredita que o PMM era formado somente por médicos estrangeiros. Provavelmente esse entendimento se deve ao fato de ter sido o alvo mais polêmico do programa e o mais explorado pela mídia ${ }^{36}$, com a publicação de artigos com posicionamentos e discussões sobre o programa, colocando em evidência a contratação de médicos estrangeiros, com ênfase na chegada dos médicos cubanos. A categoria médica foi a que mostrou maior resistência ao Programa: os estudantes e docentes de medicina desconheciam que o Programa continha outros eixos além do de provimento, sendo importante estimular o debate nas escolas médicas sobre o Programa e suas ações ${ }^{37}$.

\section{Mudanças nas práticas e modelos de saúde}

Verificou-se, nos estudos realizados, a presença de traços de integralidade nas práticas das equipes de saúde da família a partir da incorporação dos médicos do Programa nas seguintes dimensões: acessibilidade, acolhimento, vínculo, respeito aos usuários e continuidade de cuidados. Da mesma forma, verificou-se que, na ótica dos trabalhadores das equipes de saúde da família, o PMM teria contribuído para a integralidade das práticas, impactando positivamente na melhoria da $\mathrm{ABS}^{29}$.

Estudos apresentam os comentários positivos dos usuários a partir da experiência da consulta médica, seja no consultório ou no domicílio, expressando que eles foram 'respeitados'. Uma frase bem representativa desses depoimentos é a manifestação de um usuário no sentido de que 'eles (os médicos) nos tocam e não têm nojo da gente'. Outro fato que justifica o índice de satisfação dos usuários está relacionado com não haver mais necessidade de deslocamento para buscar atendimento médico ${ }^{29}$.

Esperava-se que o PMM provocasse mudanças e reflexões diversas nos municípios que fizeram adesão ao Programa. A partir das pesquisas realizadas, percebeu-se que o tensionamento deu-se menos no sentido da construção da APS e mais no âmbito da conformação da rede e nas formas de se praticar a clínica. Assim, as potencialidades do Programa ultrapassaram qualquer barreira inicial, de modo que a população tem-se mostrado vinculada às equipes compostas por médicos participantes do Programa ${ }^{38}$.

\section{Provimento e fixação}

No primeiro ano de implementação do Programa, houve o provimento de 14.168 médicos entre os municípios que aderiram ao programa. Os editais de seleção dos médicos participantes estabeleceram prioridades na contratação e na ocupação das vagas, de modo que os profissionais com registro nos Conselhos Regionais de Medicina (CRM) tiveram prioridade sobre os médicos intercambistas individuais, que, por sua vez, foram priorizados sobre os cooperados. Estes últimos não escolheram seus municípios de atuação e eram provenientes do acordo com Cuba. É relevante referir que, entre todos os médicos participante do Programa, 11.150 eram cooperados, originários de acordo firmado entre o governo brasileiro e Cuba, ou seja, representavam aproximadamente $80 \%$ do total ${ }^{39}$.

O PMM contou com a participação de médicos de 47 diferentes nacionalidades, que tiveram a seguinte alocação: os com registro no CRM, que escolheram o local de atuação, foram alocados principalmente em municípios com $20 \%$ ou mais da população vivendo em extrema pobreza (31,3\%). Um terço dos 
médicos cooperados, ou seja, aqueles oriundos do acordo entre o governo brasileiro e cubano, também foram alocados em municípios com o perfil supracitado (31,7\%). Os intercambistas individuais optaram, principalmente, por regiões metropolitanas e capitais; e, em último lugar, por municípios com alguma situação de vulnerabilidade. A região Nordeste foi a que mais recebeu médicos cooperados, seguida pelo Sudeste e pelo Sul9,40.

Verificou-se que as áreas de alocação de grande parte dos médicos brasileiros, mesmo que em áreas de maior vulnerabilidade socioeconômica, configuraram os municípios com menores distâncias das capitais. Tal fato indica, por parte dos médicos brasileiros, uma opção, predominantemente, por cidades próximas aos grandes centros urbanos ${ }^{40}$.

A possibilidade de opção de escolha, por parte dos médicos participantes do Programa, de áreas não prioritárias permitiu a permanência das desigualdades regionais no acesso à atenção em saúde ${ }^{39}$.

Apesar dos avanços no provimento de profissionais, verificou-se a insuficiência no apoio profissional e pessoal, que se caracteriza por ações de promoção a cooperação interprofissional, melhoria nas condições de trabalho, organização de planos de carreiras e salários, medidas de reconhecimento profissional e apoio ao desenvolvimento de redes de profissionais para diminuir o isolamento dos trabalhadores nas áreas que são mais remotas e vulneráveis. Diversos autores afirmam que as ações de apoio profissional e pessoal influenciam diretamente a decisão do profissional por permanecer em um local, trazendo benefícios em longo prazo; porém, no âmbito do PMM, observa-se baixo investimento financeiro nessa dimensão ${ }^{24}$.

\section{Análise da arena política e a imple- mentação do programa}

Como anteriormente mencionado, atualmente, compreende-se que a base do PMM se configurou no ano de 2012; no entanto, as manifestações das Jornadas de Junho em 2013 abriram uma janela de oportunidades para o lançamento e a implementação imediata do PMM.

Observou-se uma disputa discursiva pela legitimidade do Programa, em que os grandes meios de comunicação, inicialmente, posicionaram-se contra o PMM; e as redes sociais, com propostas alternativas de comunicação, foram relevantes no contraponto a essas posições. No mesmo sentido, houve movimentos de oposição e contestação da legitimidade e da eficiência da proposta, que tomaram as ruas, as mídias e os foros legislativos e judiciários. As entidades de classe da categoria médica foram as que mostraram maior resistência à proposta 4 . Na intensidade dos jogos políticos, houve recuo e concessão dos sujeitos sociais que apoiavam e que eram contra o programa.

Assim, o PMMB, situado no âmbito da Política do PMM, foi instituído com a finalidade de garantir o provimento de médicos em localidades carentes de profissionais e de investir na formação de médicos na área de atenção básica, mediante integração ensino-serviço, inclusive por meio de intercâmbio internacional ${ }^{35}$.

Inicialmente, foram inúmeras as polêmicas acerca de justificativas, propósitos, tipos de contratações e sobre os médicos contratados pelo programa ${ }^{10}$. Com relação à participação na formulação do Programa, em face da emergência no seu lançamento, não houve o tempo necessário para uma discussão mais ampliada entre os diferentes entes federados e a sociedade, o que prejudicou no planejamento e na divulgação da política. Aliás, a pouca participação de diferentes setores e atores sociais no processo de formulação e implementação do programa explica, em parte, as críticas sofridas na sua origem, uma vez que a fase inicial foi conduzida de maneira bastante centralizada pelo Governo Federal2,41.

Apesar das adversidades, houve o reconhecimento da população, pois o PMM apresentou uma estratégia efetiva de solução à insuficiência de médicos em algumas localidades, ampliando o acesso à assistência. No entanto, pode-se dar destaque à reação da corporação 
médica brasileira à contratação dos profissionais estrangeiros, à desinformação em relação ao Programa pela população e pela mídia, limitando a compreensão à contratação de médicos cubanos ${ }^{41}$.

Da mesma forma, existiu o reconhecimento de parte de sanitaristas e acadêmicos defendendo que o Programa permitiu o fortalecimento da atenção básica e a ampliação do acesso a serviços de saúde ${ }^{2,42}$.

\section{Direito à saúde}

No Brasil, o acesso aos serviços de Saúde no âmbito do SUS deve ser ordenado pela atenção primária; porém, a garantia do acesso é complexa, pois abrange diferentes dimensões: política, organizacional, técnica, simbólica e geográfica. Estudos realizados no município de Mossoró (Rio Grande do Norte) apresentam que, na perspectiva do usuário, com a implementação do PMM, a atenção básica se configura de maneira mais acessível no que tange à consulta médica, apesar das dificuldades estruturais referentes à prestação de um serviço de saúde oportuno e resolutivo ${ }^{\mathbf{3 4}}$.

A identificação da grande inserção de médicos do PMM sugere a atuação destes profissionais em equipes incompletas ou com elevada rotatividade, cenários esses de ofertas de serviços de saúde que não permitiam a constituição de vínculos entre usuários e trabalhadores de saúde, nem a continuidade do cuidado. É evidente que o PMM ampliou o acesso e a garantia do direito à saúde a milhões de brasileiros por meio do provimento imediato $^{26}$. Construiu uma estratégia exitosa em termos de legitimação pela própria natureza das ações propostas, ou seja, uma solução de curto prazo, alta visibilidade e percepção a uma parcela considerável da população brasileira, para quem o acesso a médico era restrito e, agora, torna-se direto e observável. Dessa forma, o programa ganha credibilidade e legitimidade perante o usuário como uma solução efetiva ${ }^{2}$.

No entanto, apesar de o PMM ter ampliado o acesso à assistência à saúde em áreas socialmente vulneráveis e remotas, pode-se observar que os municípios ainda convivem com insegurança assistencial, tendo em vista que ocorreram redução e substituição da oferta regular de médicos das prefeituras pelo provimento federal, no qual os médicos intercambistas têm um vínculo de três anos, que pode ser renovado por igual período. Esse fato coloca em questão o vínculo entre usuários e equipe de saúde ${ }^{9}$.

Não obstante, mesmo diante desse cenário, considerando o direito à cidadania e os princípios do SUS, estudos evidenciam que a implementação do PMM contribuiu para o fortalecimento da atenção básica, para a qualificação dos serviços de saúde, para a melhoria do provimento médico e para um significativo aprimoramento do acesso aos serviços de saúde à população 5 .

\section{Considerações finais}

Esta revisão expressa a significativa produção de conhecimentos referentes ao PMM, especificamente, relacionados com o eixo de provimento emergencial, denominado PMMB. Os dados demonstram um grande esforço em análises do Programa a partir dos espaços locais (municípios, estados e regiões de saúde; com poucos estudos de abrangência nacional) em que se desenvolvia essa política, o que permite a compreensão da implementação do Programa nas diferentes regiões do País.

No entanto, apesar de os estudos realizarem a análise de experiências locais, verificou-se pouca produção de conhecimentos e de pesquisas advindas dos serviços, sendo realizadas em grande parte pelas Instituições de Ensino Superior. Assim, grande parte dos estudos dão ênfase à formalidade da política, com destaque ao aspecto legal, institucional, enunciado oficial e práticas de sujeitos que elaboram e executam a política ${ }^{36}$. Finalizada a revisão, é possível afirmar que poucos estudos têm abordado a dimensão mais micropolítica do PMM, de modo a incorporar a perspectiva 
*Orcid (Open Researcher and Contributor ID). de seus protagonistas centrais.

Percebeu-se, neste estudo, o interesse de diferentes disciplinas e áreas de conhecimento na abordagem e na reflexão dessa Política, o que permite uma reflexão dialogada entre os diferentes estudos na busca de desenvolvimento de caminhos para uma análise crítica da Política em curso, rompendo com a ideia de que apenas um campo específico

\section{Referências}

1. Craveiro I, Hortale V, Oliveira APC, et al. The utilization of research evidence in Health Workforce Policies: the perspectives of Portuguese and Brazilian National Policy-Makers. j. public health.2018; 40(Supl1):i50-i56.

2. Lotta GS, Galvão MCCP, Favareto AS. Análise do Programa Mais Médicos à luz dos arranjos institucionais: intersetorialidade, relações federativas, participação social e territorialidade. Ciênc. Saúde Colet. [internet]. 2016 [acesso em 2018 set 1]; 21(9):27612772. Disponível em: http://dx.doi.org/10.1590/141381232015219.16042016.

3. Souza Lima RCG, Garcia Junior CAS, KerkorskI E, et al. Programa Mais Médicos no Estado de Santa Catarina: breve relato de experiências iniciais. Tempus (Brasília) [internet]. 2016 [acesso em 2018 set 1]; 9(4):67-80. Disponível em: http://www.tempusactas. unb.br/index.php/tempus/article/view/1741.

4. Macedo AS, Alcântara VC, Andrade LFS, et al. O papel dos atores na formulação e implementação de políticas públicas: dinâmicas, conflitos e interesses no Programa Mais Médicos. Cad. EBAPE.BR [internet]. 2016 [acesso em 2018 set 1]; 14(esp):593-618. Dispo- tem capacidade de construir conhecimentos sobre Política ${ }^{\mathbf{3}}$.

\section{Colaboradores}

Silva QTA (0000-0001-5464-6307)* e Cecílio LCO (0000-0002-9207-4781)* contribuíram, igualmente, na elaboração do manuscrito. nível em: http://dx.doi.org/10.1590/1679-395117188.

5. Mota RG, Barros NF. O Programa Mais Médicos no Estado de Mato Grosso, Brasil: uma análise de implementação. Ciênc. Saúde Colet. [internet]. 2016 [acesso em 2018 set 1]; 21(9):2879-2888. Disponível em: http:// dx.doi.org/10.1590/1413-81232015219.14582016.

6. Brasil. Exposição de Motivo no 00024/2013 de 6 de julho de 2013. Medida Provisória que institui o Programa Mais Médicos [internet]. [acesso em 2018 set 1]. Disponível em: http://www.planalto.gov.br/ccivil_03/_Ato2011-2014/2013/Mpv/mpv621.htm.

7. Oliveira FP, Vanni T, Pinto HA, et al. Mais Médicos: um programa brasileiro em uma perspectiva internacional. Interface (Botucatu) [internet]. 2015 [acesso em 2018 set 1]; 19(54):623-634. Disponível em: http:// dx.doi.org/10.1590/1807-57622014.1142.

8. sil: dados gerais e descrições de desigualdades [internet]. 2011 [acesso em 2018 set 1]; São Paulo: Conselho Regional de Medicina do Estado de São Paulo, Conselho Federal de Medicina. Disponível em: https://www. cremesp.org.br/pdfs/demografia_2_dezembro.pdf. 
9. Girardi SN, Van Stralen ACS, Cella JN, et al. Impacto do Programa Mais Médicos na redução da escassez de médicos em Atenção Primária à Saúde. Ciênc. Saúde Colet. [internet]. 2016 [acesso em 2018 set 1]; 21(9):2675-2684. Disponível em: http://dx.doi. org/10.1590/1413-81232015219.16032016.

10. Miranda AS, Melo DA. Análise comparativa sobre a implantação do Programa Mais Médicos em agregados de municípios do Rio Grande do Sul, Brasil. Ciênc. Saúde Colet. [internet]. 2016 [acesso em 2018 set 1]; 21(9):2837-2848. Disponível em: http://dx.doi. org/10.1590/1413-81232015219.18522016.

11. Dal Poz MR, Sepulveda HR, Costa Couto MH, et al. Assessment of human resources for health programme implementation in 15 Latin American and Caribbean countries. Hum Resour Health. 2015;13-24.

12. Pan American Health Organization. Toronto Call to Action: 2006-2015 towards a decade of human resources for health in the Americas [internet]. Washington, DC; WHO; 2006. [acesso em 2018 ago 18]. Disponível em: http://www.bvsde.paho.org/bvsdeescuelas/fulltext/CallAction_eng1.pdf.

13. Roas AC. Experimentos de escolha discreta sobre provimento e fixação de recursos humanos em saú-

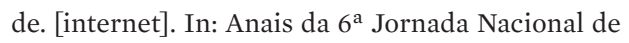
Economia da Saúde; 2012; Brasília, DF: Associação Brasileira de Economia da Saúde; 2012 [acesso em 2018 set 1]. Disponível em: http://abresbrasil.org.br/ sites/default/files/poster_21_0.pdf.

14. Wismar M, Maier CB, Glinos IA, et al, editores. Health professional mobility and health systems: evidence from 17 European countries. London: European Observer. 2011.

15. Willis-Shattuck M, Bidwell P, Thomas S, et al. Motivation and retention of healthworkers in developing countries: a systematic review. BMC Health Serv Res. 2008; 8:247-255.

16. Garcia ACP. Gestão do trabalho e da educação na saúde: uma reconstrução histórica e política [tese] [internet]. Rio de Janeiro: Universidade do Estado do Rio de Janeiro, 2010 [acesso em 2018 set 1]. Disponível em: http://www.obsnetims.org.br/uploaded/2_5_2013_0_Gestao_trabalho.pdf.

17. Organización Mundial de la Salud. Argumentando sobre la cobertura sanitária universal Ginebra: OMS; 2013.

18. Organización Naciones Unidas. Resolución 70/1 de 25 de septiembre de 2015. Transformar nuestro mundo: la Agenda 2030 para el Desarrollo Sostenible. Assembleia Geral. 21 Out 2015.

19. Brasil. Ministério da Saúde. Secretaria de Gestão do Trabalho e da Educação na Saúde. Seminário nacional sobre escassez, provimento e fixação de profissionais de saúde em áreas remotas de maior vulnerabilidade. Brasília, DF: Ministério da Saúde; 2012.

20. Dal Poz M. Entre o prescrito e o realizado: estudo sobre a implantação do SUS no Estado do Rio de Janeiro e sua repercussão na política de recursos humanos em nível municipal [tese]. Rio de Janeiro: Universidade do Estado do Rio de Janeiro, 1996. 176 p.

21. Pierantoni CR. Reformas da saúde e recursos humanos: novos desafios x velhos problemas [tese] [internet]. Rio de Janeiro: Universidade do Estado do Rio de Janeiro, 2010 [acesso em 2018 mar 5]. Disponível em: http://www.obsnetims.org.br/uploaded/3_6_2013_0_Reformas_da_saude.pdf.

22. Organização Pan-Americana da Saúde. A atenção à saúde coordenada pela APS: construindo as redes de atenção no SUS - Contribuições para o debate. Brasília, DF: OPAS; 2011

23. Viana ALD, Dal Poz MR. A reforma do sistema de saúde no Brasil e o Programa de Saúde da Família. Physis [internet]. 1998 [acesso em 2018 set 1]; 8(2):1148. Disponível em: http://dx.doi.org/10.1590/S010373311998000200002 .

24. Carvalho VKS, Marques CP, Silva EN. A contribuição do Programa Mais Médicos: análise a partir das recomendações da OMS para provimento de médicos. Ciênc. Saúde Colet. [internet]. 2016 [acesso em 
2018 set 1]; 21(9):2773-2784. Disponível em: http:// dx.doi.org/10.1590/1413-81232015219.17362016.

25. Campos CVA, Malik AM. Satisfação no trabalho e rotatividade dos médicos do Programa de Saúde da Família. Rev. Adm. Pública [internet]. 2008 [acesso em 2018 set 1]; 42(2):347-368. Disponível em: http:// dx.doi.org/10.1590/S0034-76122008000200007.

26. Giovanella L, Mendonça MHM. Atenção Primária à Saúde. In. Giovanella L, Escorel S, Lobato LVC, et al. Políticas e Sistema de Saúde no Brasil. Rio de Janeiro: Fiocruz; 2012. p. 493-545.

27. Vosgerau DSAR, Romanowsi JP. Estudos de revisão: implicações conceituais e metodológicas. Rev. Diálogo Educ. 2014; 14(41):165-189.

28. Fonseca JJS. Referências para a elaboração de um artigo de pesquisa. SlideShare [internet]. [acesso em 2018 set 1]. Disponível em: http://www.slideshare. net/joaojosefonseca/referncias-para-aelaborao-de-um-artigo-de-pesquisa.

29. Comes Y, Trindade JS, Pessoa VM, et al. A implementação do Programa Mais Médicos e a integralidade nas práticas da Estratégia Saúde da Família. Ciênc. Saúde Colet. [internet]. 2016 [acesso em 2018 set 1]; 21(9):2729-2738. Disponível em: http://dx.doi. org/10.1590/1413-81232015219.15472016.

30. Carneiro VB, Oliveira PTR, Bastos MSCB, et al. Avaliação da mortalidade e internações por Condição Sensível à Atenção Primária em menores de 5 anos, antes e durante o Programa Mais Médicos, no Marajó-Pará-Brasil. Saúde Redes [internet]. 2016 [acesso em 2018 set 1]; 2(4):360-371. Disponível em: http:// revista.redeunida.org.br/ojs/index.php/rede-unida/ article/view/778.

31. Gonçalves RF, Sousa IMC, Tanaka OY, et al. Programa Mais Médicos no Nordeste: avaliação das internações por condições sensíveis à Atenção Primária à Saúde. Ciênc. Saúde Colet. [internet]. 2016 [acesso em 2018 set 1]; 21(9):2815-2824. Disponível em: http://dx.doi. org/10.1590/1413-81232015219.15392016.
32. Carrer A, Toso BRGO, Guimarães ATB, et al. Efetividade da Estratégia Saúde da Família em unidades com e sem Programa Mais Médicos em município no oeste do Paraná, Brasil. Ciênc. Saúde Colet. [internet]. 2016 [acesso em 2018 set 1]; 21(9):28492860. Disponível em: http://dx.doi.org/10.1590/141381232015219.16212016

33. Santos M, Souza E, Cardoso J. Avaliação da Qualidade da Estratégia Saúde da Família e do Programa Mais Médicos na Área Rural de Porto Velho, Rondônia. Gest. Sociedade [internet]. 2016 [acesso em 2018 set 1]; 10(26):1327-1346. Disponível em: https:// www.gestaoesociedade.org/gestaoesociedade/article/view/2089.

34. Silva TRB, Silva JV, Pontes AGV, et al. Percepção de usuários sobre o Programa Mais Médicos no município de Mossoró, Brasil. Ciênc. Saúde Colet. [internet]. 2016 [acesso em 2018 set 1]; 21(9):28612869. Disponível em: http://dx.doi.org/10.1590/141381232015219.18022016.

35. Thumé E, Wachs LS, Soares MU, et al. Reflexões dos médicos sobre o processo pessoal de aprendizagem e os significados da especialização à distância em saúde da família. Ciênc. Saúde Colet. [internet]. 2016 set [acesso em 2018 set 1]; 21(9):2807-2814. Disponível em: http://dx.doi.org/10.1590/1413-81232015219.14632016.

36. Baião DC, Leônidas SR, Lins CFM. Avaliação do Programa Mais Médicos: uma revisão da literatura [internet]. In: XIV Encontro de Pós-Graduação e Pesquisa; 2014 Out 20-24; Fortaleza: Universidade de Fortaleza [acesso em 2018 set 1]. Disponível em: http://uol. unifor.br/oul/conteudosite/?cdConteudo=5009022.

37. Villa Real GGO, Succi GM, Montalli VAM, et al. Estudantes e Professores da Área da Saúde Conhecem o Programa Mais Médicos? Rev. bras. educ. med. [internet]. 2017 [acesso em 2018 set 1]; 41(1):110116. Disponível em: http://dx.doi.org/10.1590/1981$-52712015 v 41 n 1 r b 20160036$.

38. Terra LSV, Borges FT, Lidola M, et al. Análise da experiência de médicos cubanos numa metrópole brasileira segundo o Método Paideia. Ciênc. Saúde Colet. 
[internet]. 2016 [acesso em 2018 set 1]; 21(9):28252836. Disponível em: http://dx.doi.org/10.1590/141381232015219.15312016 .

39. Oliveira JPA, Sanchez MN, Santos Leonor MP. O Programa Mais Médicos: provimento de médicos em municípios brasileiros prioritários entre 2013 e 2014. Ciênc. Saúde Colet. [internet]. 2016 [acesso em 2018 set 1]; 21(9):2719-2727. Disponível em: http://dx.doi. org/10.1590/1413-81232015219.17702016.

40. Nogueira PTA, Bezerra AFB, Leite AFB, et al. Características da distribuição de profissionais do Programa Mais Médicos nos estados do Nordeste, Brasil. Ciênc. Saúde Colet. [internet]. 2016 [acesso em 2018 set 1]; 21(9):2889-2898. Disponível em: http://dx.doi. org/10.1590/1413-81232015219.17022016.
41. Carryl JAM, Rossato L, Prados RMN. Programa Mais Médicos. Rev. Diálogos Interdisciplinares. 2016; 5(2):1-8.

42. Pinto HA, Sales MJT, Oliveira FP, et al. O Programa Mais Médicos e o fortalecimento da Atenção Básica. Divulg. Saúde debate. 2014; 51:105-120.

43. Mattos RA, Baptista TWF, organizadores. Caminhos para análise das políticas de saúde. Porto Alegre: Rede Unida; 2015.

Recebido em 14/09/2018

Aprovado em 02/04/2019

Conflito de interesses: inexistente

Suporte financeiro: não houve 